\title{
Elevated Myocardial VCAM1 Expression is Associated with Higher Risk of Dilated Cardiomyopathy or Ischemic Heart Disease-caused Heart Failure and Higher Immune Infiltration in Failing Myocardium
}

\section{Tongyu Wang}

The Fourth Affiliated Hospital of China Medical University

Jiahu Tian

The Fourth Affiliated Hospital of China Medical University

Yuanzhe Jin ( $\nabla$ yzjin@cmu.edu.cn )

The Fourth Affiliated Hospital of China Medical University

\section{Research Article}

Keywords: immune cell infiltration, VCAM1, IHD, DCM, heart failure, bioinformatic analysis

Posted Date: July 7th, 2021

DOI: https://doi.org/10.21203/rs.3.rs-658306/v1

License: (1) (i) This work is licensed under a Creative Commons Attribution 4.0 International License. Read Full License 


\section{Abstract}

Background. Ischemic heart disease (IHD) and dilated cardiomyopathy (DCM) are the two most common etiologies of heart failure (HF). Both forms share common characteristics including ventricle dilation in the final stage. Immune mechanisms in HF are increasingly highlighted and have been implicated in the pathogeneses of IHD and DCM. A better understanding of adhesion molecule expression and correlated immune cell infiltration could enhance disease detection and improve therapeutic targets.

Objective. This study was performed to explore the common mechanisms underlying IHD and DCM.

Methods. After searching the Gene Expression Omnibus database, we selected the GSE42955 and GS57338 datasets for analysis, which contain 29 and 313 samples, respectively.

Results. The screening stage revealed that vascular cell adhesion molecule 1 (VCAM1) and intercellular adhesion molecule 1 (ICAM1) play pivotal roles in regulating DEGs. Subsequent analyses revealed that VCAM1 was differentially expressed in the myocardium and involved in regulating immune cell infiltration. We also found that dysregulated VCAM1 expression was associated with a higher risk of HF by constructing a clinical risk-predicting model.

Conclusions. Collectively, our results suggest that VCAM-1 could be used as a biomarker or therapy target for HF.

\section{Introduction}

Heart failure (HF) is a clinical syndrome characterized by fatigue, dyspnea, and fluid retention. It is usually caused by left or whole-heart systolic dysfunction and is accompanied by congestion (Bergethon et al. 2016). Due to the growing aging population and increasing prevalence rates of risk factors including hypertension, diabetes, and obesity, the global prevalence of HF is on the rise. The Rotterdam study showed that after adjustment for age, HF patients had a two-fold higher risk of total mortality compared with control subjects, and the risk of sudden death was four to six times higher (Mosterd et al. 2001).

Ischemic heart disease (IHD) and dilated cardiomyopathy (DCM) are the main causes of HF. Both have clinical manifestations of cardiac insufficiency and overlapping symptoms and signs, but they lack specific manifestations. DCM is mainly characterized by non-ischemic left ventricular expansion accompanied by changes in cardiac structure and function, and it is the most prevalent cause of chronic congestive HF in individuals between the ages of 20 and 60 years old (Elliott 2000; Qu et al. 2015). Ventricular structure and function can change due to genetic variation, infection, inflammatory responses, and autoimmune disease. Therefore, the American Heart Association classifies DCM as inherited, mixed, or acquired according to its etiology. Decades of research have shown that genetic variations are the main etiology of DCM (Schultheiss et al. 2019). Most HF due to DCM ( 70\%) is the result of decreased myocardial contractive force caused by ventricular dilatation, whereas IHD causes chronic ventricular 
remodeling that eventually also causes ventricular dilatation and then HF (Eckhouse et al. 2013). This suggests that both conditions may share an underlying mechanism that causes HF. During the last decades, microarray technology and bioinformatic analyses have been widely used to screen genetic alterations at the genome level, which have helped us identify differentially expressed genes (DEGs) and functional pathways involved in the pathogeneses of many diseases (Barrett et al. 2013). Searching the Gene Expression Omnibus (GEO), we selected the GSE42955 and GSE57338 gene sets for further analysis because they are derived from myocardium array data. The results revealed that vascular cell adhesion molecule 1 (VCAM1) was abnormally expressed in both DCM and IHD patients. Based on this, we speculated that this protein plays an important role in the development of both conditions and that it could be a useful biomarker for the prognostic assessment of patients with HF. The goal of this study was to further explore the utility of VCAM1 as a biomarker in HF induced by DCM and IHD.

Studies have implicated chronic inflammation in the development of myocardial structural and functional abnormalities during HF pathogenesis (Tousoulis et al. 2001). Inflammatory biomarkers play an important role in the prognostic assessment of patients with HF. For example, Alonso-Martinez et al. showed that patients with acute HF are at higher risk of hospitalization when their C-reactive protein (CRP) level is $>9 \mathrm{mg} / \mathrm{L}^{-1}$, and CRP level is also associated with HF severity. VCAM1 is an adhesion molecule expressed on the activated endothelial surface; it promotes leukocyte adhesion and crossepithelial migration by binding to leukocyte ligands to promote the inflammatory response (AlonsoMartínez et al. 2014). VCAM1 expression is significantly increased in patients with HF caused by acute myocardial infarction and has good predictive value for patient prognosis (Savic-Radojevic et al. 2013). Michowitz et al. showed that VCAM1 mediated the production of reactive oxygen species (ROS) by NADPH oxidase and further activated matrix metalloproteinases to induce ventricular remodeling (Michowitz et al. 2008). The myocardium can be affected by numerous pathophysiological processes that can be broadly classified as ischemic and non-ischemic. In terms of the global burden of disease, ischemic injury is the main pathophysiological mechanism of myocardial injury. Irreversible HF often occurs following acute ischemic injury or progressive impairment of cardiac function due to various clinical pathological causes (Jacoby and McKenna 2012). When the myocardium becomes ischemic, the death of damaged and necrotic cardiomyocytes leads to tissue-resident immune and non-immune cell activation. Expansion of neutrophil and macrophage populations is responsible for the removal of dead cells and matrix debris, as well as the release of cytokines and growth factors that stimulate formation of highly vascularized granulation tissue (i.e., connective tissue and new vasculature) (Frangogiannis 2006). Pro-inflammatory cytokines and chemokines produced by immune cells can recruit inflammatory white blood cells from the bloodstream into damaged areas (Frangogiannis and Entman 2005). The immune system drives acute inflammatory and regenerative responses after heart tissue damage (Hofmann et al. 2012), and immune cells are involved in heart damage, ischemia, inflammation, and repair (Kazuaki et al. 2010). While it is known that the immune system plays an important role in heart injury pathogenesis, more research is needed to identify the specific mechanisms (Aran et al. 2017). This study investigated the influence of VCAM1 on immune infiltration and HF occurrence and assessed the prognostic impact of VCAM1 by building an HF risk prediction model. 


\section{Methods}

\section{Acquirement of array data}

The GSE42955 and GSE57338 gene expression profiles were extracted from the GEO database.

GSE42955 was based on the GPL6244 platform (Affymetrix Human Gene 1.0 ST Array [transcript (gene) version]). The GSE42955 cohort comprised 29 samples, including 12, 12, and 5 heart apex tissue samples from DCM patients, IHD patients, and healthy controls, respectively. The GSE57338 dataset was based on the GPL11532 platform (Affymetrix Human Gene 1.1 ST Array [transcript (gene) version]). The GSE57338 cohort comprised 313 samples including the cardiac muscle (ventricle tissue) of 177 patients with HF and 136 healthy controls.

\section{DEG screen}

We screened DEGs between patients and controls using the "limma" R package (limma powers differential expression analyses for RNA-sequencing and microarray studies). The significance analysis of microarrays was utilized to select significantly different genes with $p<0.05$ and $\log _{2}$ fold change (FC) $\geq 1$.

\section{Integration of protein-protein interaction (PPI) network and the core functioning gene selection}

DEGs were mapped onto the STRING (Search Tool for the Retrieval of Interacting Genes) database (version 9.0) to evaluate inter-DEG relationships via PPI information (http://string-db.org). Protein interaction relationship networks were mapped using Cytoscape software, which analyzed the relationship between candidate DEGs encoding proteins in the cardiac muscle of patients with HF. The cytoHubba plugin was employed to identify core molecules in the PPI network, and cytoHubba scores were used to identify the hub-genes.

\section{Establishment of the clinical risk prediction model}

The genes showing correlations with VCAM1 in the Spearman correlation results were further filtered by a LASSO model. The glmnet package in R software was used with the "family" parameter=binomial, which is suitable for a logistic model. Then the cv.glmnet function of the glmnet package was used to identify the suitable lambda value for candidate genes to establish a risk prediction model. The nomogram function in the rms package was used to plot the nomogram.

\section{Immune and stromal cells analyses}


The novel gene signature-based method xCell (http://xCell.ucsf.edu/) was used to investigate 64 immune and stromal cell types with extensive in silico analyses that were also compared with cytometry immunophenotyping (Aran et al. 2017). By applying xCell to the microarray data and using the Wilcoxon method to assess variance, the estimated proportions of immune and stromal cell types were obtained for each myocardium tissue sample using a cut-off value of $p<0.05$. Cell types were categorized into lymphoid, myeloid, stromal, stem cells, and others.

\section{Gene set enrichment analysis (GSEA)}

To further explore the potential function of these genes in HF, samples in the GSE57338 dataset were divided into HF and control groups prior to GSEA (Subramanian et al. 2005). We selected Kyoto Encyclopedia of Genes and Genomes (KEGG) pathways associated with immuno-filtration which are associated with the occurrence of heart failure. We also subdivided the samples according to VCAM1 expression level (high and low expression groups) and carried out GSEA again. The R software "clusterprofiler" package was utilized to conduct the GSEA. The "c2.cp.kegg.v7.1.symbols.gmt_gsvascore," "c5.go.bp.v7.2.symbols.gmt" was selected as the reference gene set, and p-adjusted< 0.05 was chosen as the cut-off criterion.

\section{Results}

\section{DEGs in the GSE42955 gene set and hub gene selection}

The micro-array data in the GSE42955 dataset was divided into two groups (DCM vs Control and IHD vs control) prior to DEG analysis. With $\log _{2}$ fold change $(F C)=1$ as the threshold and $p>0.05$ as the standard, a total of 41 DEGs were selected in the DCM vs Control cohort (21 up-regulated and 20 down-regulated, Figure $1 \mathrm{a}$ and $1 \mathrm{~b}$ ). A total of 41 DEGs were selected in the IHD vs Control cohort (10 up-regulated and 31 down-regulated, Figure $1 \mathrm{c}$ and $1 \mathrm{~d}$ ). Then, the intersection of the screened genes was identified, and 25 common DEGs were selected (Figure 1E). The common DEGs were uploaded to the STRING database to form a network of gene interactions (Figure 1f). The core functional modules were identified using the cytoHubba plug-in for Cytoscape software. VCAM1 and intercellular adhesion molecule 1 (ICAM1) had the highest connectivity score (Figure 1g).

\section{Screening of DEGs in the GSE57338 dataset and clinical risk prediction model construction}

In the GSE57338 dataset, DEGs in heart tissues of the HF group ( $n=177)$ and non-HF control group $(n=136)$ were analyzed. Fifty DEGs were selected with $\log _{2} F C=1$ and $p>0.05$ as the thresholds (Figure 2ab). VCAM1 expression was significantly higher in the HF group, suggesting that it could be a biomarker for HF occurrence and development (Figure 2c). Spearman correlation analysis was subsequently 
performed on the DEGs identified from the GSE57338 dataset, and 34 DEGs related to VCAM1 expression were selected (Figure 2d). These 34 DEGs were then used to construct the clinical risk prediction model. Variables were screened through least absolute shrinkage and selection operator (LASSO) regression (Figure 2e-f), and 12 DEGs were finally selected for model construction (Figure $2 \mathrm{~g}$ ) because the number of samples occurring events should be 10 times of the number of variants with lambda $=0.005218785$. The Brier score was 0.033 (Figure 2h) and the final model C index was 0.987. The model showed good degrees of differentiation and calibration. The expression of the final risk score was as follows: riskscore $=-1.064 * F C N 3-0.564 *$ SLCO4A1-0.316*IL1RL1-0.124*CYP4B1-

$0.919 * \mathrm{COL} 14 \mathrm{~A} 1+1.20 * \mathrm{SMOC} 2+0.494 * \mathrm{FI} / 44 \mathrm{~L}+0.474 * \mathrm{PHLDA} 1+2.72 *$

MNS1+1.52*FREM1+0.164*C6+0.561*HBA1. In addition, a new validation cohort was established by merging the GSE5046, GSE57338, and GSE76701 datasets to validate the effectiveness of the risk model. Principal Component Analysis (PCA) results are shown in Supplementary Figure S1a-b. The Brier score in the validation cohort was 0.03 (Figure $2 \mathrm{i}$ ) and the final model $\mathrm{C}$ index was 0.984 , which demonstrate that this model has good performance in predicting the risk of HF.

Based on VCAM1 expression levels, the samples in GSE57338 were further divided; those with levels higher and lower than the median VCAM1 expression value were placed in the high- and low-expression group, respectively. By comparing the risk scores predicted by the above model between the two groups, it can be seen that the high-expression VCAM1 group had a greater risk of developing HF (Figure 2j-k).

\section{Immunoinfiltration analysis for the GSE57338 dataset}

The immunoinfiltration analysis of HF and normal myocardial tissue was performed using the xCell database, and the infiltration degree of 64 immune-related cell types was analyzed. The results for lymphocyte, myeloid immune cell, and stem cell infiltration are shown in Figure 3a-c. The infiltration of stromal cells and other cells are shown in Supplementary Figure S1. Most T lymph cells in the HF of myocardial tissue showed higher degrees of infiltration, including $C D 4^{+}$memory $\mathrm{T}$ cells, $\mathrm{CD} 4^{+}$naive $\mathrm{T}$ cells, $\mathrm{CD} 4^{+} \mathrm{T}$ cells, $\mathrm{CD} 8^{+}$naive $\mathrm{T}$ cells, natural killer (NK) cells, and $\mathrm{CD} 8^{+} \mathrm{T}$ cells. The infiltration degrees of myeloid immune cells like mast cells, conventional dendritic cells (DCs), and plasmacytoid DCs also showed increasing trends. We subsequently explored the influence of VCAM1 on immune infiltrates. As shown in Figure 3d, VCAM1 expression positively correlated with central memory $T(\mathrm{Tcm})$ cells, CD4 CD8 T cells, CD8 naive T cells, conventional DCs, and common myeloid progenitor (CMP) cells, which were significantly elevated in the HF group. Conversely, M1 macrophages, myeloid stem cells, and Th1 cells showed negative correlations with VCAM1 expression, and those cell types showed lower infiltration in the HF group. This suggests that higher VCAM1 expression increased the risk of HF by influencing the degree of immune cell infiltration. Using clusterprofiler packages, we next explored immune pathway enrichment by performing separate GSEAs in the HF and control groups and high and low VCAM1 expression groups. The HF group showed obvious enrichment of immune infiltration-related pathways (Figure 3e-f). Subsequent Gene Ontology (GO) Biological Process (BP) enrichment analyses showed enrichment of BPs related to immune cell activation and differentiation in the high VCAM1 group and HF 
groups(Figure 3g-h). Collectively, these findings indicate that VCAM1 is associated with a higher degree of immune infiltration, which is often associated with an increased risk of HF.

\section{Discussion}

$\mathrm{HF}$ is a chronic heart syndrome; most patients live for an average of 5 years after diagnosis, and more than 25 million people are currently at risk of death worldwide. HF begins with pathological heart remodeling, in which the left ventricle and other cardiac chambers develop progressive structural and functional abnormalities in response to pathological stress (MD 2013). IHD and DCM are two important etiologies of HF (Tomasoni et al. 2019). The main manifestation of HF caused by DCM is ventricular enlargement, while IHD leads to decreased myocardial cell viability and increased ROS production due to continuous myocardial ischemia. ROS can directly act on cell membranes and induce myocardial cell apoptosis, resulting in decreased cardiac output. The gradual increase of cardiac load leads to ventricular remodeling, the final stage of which is ventricular dilation leading to HF. The pathways and molecules that differ between IHD and DCM and the mechanisms by which they cause HF have been explored (Sweet et al. 2018). However, there is a paucity of studies exploring the common pathways and molecules between the two HF etiologies.

This investigation employed bioinformatics methods using the GSE42955 and GS57338 datasets to screen DEGs shared by IHD and DCM. We then established an interaction network, and the analysis showed that the VCAM1 and ICAM1 genes showed the highest connectivity. Previous studies have shown that patients with HF have significantly higher levels of ICAM1 and VCAM1 compared to controls, and elevated VCAM1 was expression associated with HF severity (Tousoulis et al. 2001). We therefore set out to explore whether there is differential expression of VCAM1 and ICAM1 in failing heart tissue. Analysis of myocardial levels of VCAM1 and ICAM1 in the HF and control groups of the GSE57338 dataset showed that only VCAM1 was significantly differentially expressed. Correlation analysis between DEGs in the HF group and VCAM1 expression was conducted to identify genes with significant correlations. We propose that the expression of these genes was controlled by VCAM1. Finally, we established a risk prediction model with the genes correlated with VCAM1 expression. The subsequent analysis showed that the risk of HF increased with higher VCAM1 levels.

VCAM1 is an adhesion molecule; activation of this endothelial surface ligand enhances binding with white blood cells, thereby increasing leukocyte adhesion and epithelial cell migration (Wrigley et al. 2013). Experimental studies have shown that immune response mechanisms are correlated to pathologic heart remodeling, causing left ventricular dysfunction and eventually leading to HF. Based on this, we explored the relationship between VCAM1 and myocardial immune infiltration and examined how VCAM1 affects infiltrating immune cells and the subsequent effect on HF risk (Zhang et al. 2017). The xCell algorithm was used to predict the infiltration degree of various immune cells in cardiac tissue, and correlation analysis was conducted to assess the relationship between VCAM1 expression and the infiltration degrees of various immune cells. The results showed that VCAM1 level was positively correlated with the numbers of CD8 T cells, CD8 Tcm cells, CD4 naive T cells, conventional DCs, CMPs, and other immune 
cells. Notably, these cells showed higher degrees of immune infiltration degree in HF tissue compared to normal tissue. Previous studies have shown that infiltrated monocytes in the myocardium can differentiate into macrophages and promote tissue damage repair (Rhee and Lavine 2020). As highly specific antigen-presenting cells involved in adaptive and innate immunity, DCs also play an important role in the occurrence of HF. Animal experiments revealed that exogenous DCs can induce autoimmune inflammation mediated by $\mathrm{CD} 4+\mathrm{T}$ cells to promote ventricular dilation and HF (Eriksson et al.). Increased T lymphocyte infiltration, which is involved in adaptive immunity, was also associated with HF risk (Strassheim et al. 2019). One of the most important features of chronic HF is the presence of numerous mature T cell infiltrates in the myocardial tissue (Moro-García et al. 2018; Ngwenyama et al. 2019). Animal studies have shown that T-cell-deficient mice are less likely to develop HF after aortic ligation (Gröschel et al. 2018), and remodeling of T cell subsets promotes HF development as indicated by elevated brain natriuretic peptide levels (Laroumanie et al. 2014). In vitro experiments revealed that Th1 cells-an important subset of T cells-can release interferon-g to promote the transformation of myocardial fibroblasts into a-smooth muscle actin fibroblasts, which can promote myocardial fibrosis, an important ventricular remodeling process (Travers et al. 2016). Therefore, $T$ cells and their subsets play important roles in HF occurrence and pathogenesis (Nevers et al. 2017). Myeloid immune cells are the most abundant immune cells in myocardium. Immune cells in healthy subjects do not produce harmful chronic inflammation under physiologic conditions, but in pathological conditions such as acute or chronic ischemia, the degree of myeloid immune cell infiltration in the myocardium increases, and they release a variety of inflammatory mediators that stimulate chronic fibrosis and remodeling to exacerbate HF (Matthias 2018). The results of this study revealed an increased degree of infiltration of myeloid progenitors and cells in HF tissues. This was positively correlated with the expression of VCAM1 that can stimulate their differentiation macrophages and monocytes. Moreover, an uncontrolled inflammatory response in the pathological state triggers a large number of monocytes to differentiate into macrophages and cause tissue damage, and extensive monocyte infiltration in cardiac tissue has been associated with increased risk of HF (Shahid et al. 2018). Because most immune cells are recruited from the blood, as an adhesion factor expressed on the vascular endothelium, VCAM1 can recruit myeloid progenitor cells to infiltrate the myocardium and differentiate into various subsets of myeloid immune cells to promote HF (Sager et al. 2016). In our study, VCAM1 expression was positively correlated with the infiltration of these immune cells, which led us to hypothesize that the increased risk of HF caused by elevated VCAM1 expression was associated with VCAM1 regulation of immune cell infiltration.

We also conducted a GSEA of immune-infiltration-related KEGG pathways comparing HF and normal tissues and high- and low-VCAM1 expression groups. The results showed that immune-related pathways were enriched in both HF and myocardial tissues with increased VCAM1 expression, and the enrichments included graft-versus-host- and Th17 differentiation-related signaling pathways. The proportion of Th17 cells in the blood circulation and the amounts of cytokines they secrete are reportedly increased in patients with HF (Myers et al. 2016). In addition, Th17 cell differentiation often requires transforming growth factor-b and interleukin (IL)-6, which are involved in myocardial fibrosis development. In addition, IL-23 secreted by Th17 cells can promote the secretion of granulocyte-macrophage colony-stimulating 
factor by Th17 cells, infiltration of other immune cells, and a chronic inflammatory response (El-Behi et al. 2011). An increase in Th17 cells is often accompanied by a decrease in Treg cells (Lu et al. 2020), which is consistent with the results observed in this study. Therefore, we propose that VCAM1 promotes HF by regulating Th17 cell infiltration. We also observed that autoimmune-related graft-versus-host and xenograft rejection pathways were significantly enriched in the myocardial tissues of patients with HF and subjects with increased VCAM1 expression, and the autoimmune response is one of the important mechanisms for HF occurrence and development (Marty et al. 2006). B-cell pathways were also enriched in HF tissues and myocardial tissue with increased VCAM1 expression, and B-cell activation has been associated with the production of autoimmune antibodies (Hofmann et al. 2018). Cytotoxic pathways of NK cells that play a role in graft immune rejection and cause cell damage through direct contact with graft cells (Gill and Lin 2019) were also enriched in our results. Based on our observation of more infiltrated NK cells in the myocardial tissue of patients with HF, VCAM1 may regulate the cytotoxicity of NK cells and promote myocardial injury by participating in related signaling pathways. On the other hand, GSEA revealed that functions associated with $\mathrm{T}$ and $\mathrm{B}$ cell activation were obviously enriched in HF patients and subjects with high VCAM1 expression, providing additional evidence that VCAM1 is related to the regulation of immune cell infiltration in HF.

We also established a risk model of HF that showed good performance in the training and validation cohorts. This could benefit clinical application in predicting the risk of HF and serve as single biomarker in risk prediction. With regard to HF therapies, there have been limited studies on those targeting VCAM1, and our results may provide evidence for future treatments.

\section{Limitations}

This study established a prediction model, however the biomarkers involved in the model were not discussed, and the selection was based on Spearman correlations alone, which neglects functional correlation. However, the aim of model establishment was to calculate the risk score of each sample and see the difference between VCAM1 high- and low-expression groups. Although studies have investigated the association between VCAM1 and HF, most focused on circulating VCAM1 levels. We examined dysregulated VCAM1 expression in myocardium tissue, which may be more clinically meaningful. In addition, we investigated immune cell infiltration in myocardium and explored the connection to VCAM1 dysregulation, which has not been reported previously.

\section{Conclusions}

VCAM1 can be considered a useful biomarker for high risk for HF. The protein could function through immune infiltration by regulating or participating in the process of immune cells working in heart injury or repair. We established a clinical risk prediction model involving DEGs that could be also used to evaluate the risk for HF.

\section{Declarations}




\section{Acknowledgements}

Thanks for our country for providing us this opportunity to do this research work.

\section{Author contributions}

T.W. and J.T. wrote the main manuscript text and T.W prepared figures 1-3. All authors reviewed the manuscript.

\section{Competing interests}

The authors declare no competing interests.

\section{Data availability}

We provide the raw data in Supplementary files.

\section{References}

1. Alonso-Martínez, J. L. et al. C-reactive protein as a predictor of improvement and readmission in heart failure. Eur. J. Heart Fail, 4, 331-336 (2014).

2. Aran, D., Hu, Z. \& Butte, A. J. xCell: digitally portraying the tissue cellular heterogeneity landscape. Genome Biol, 18, 220 (2017).

3. Barrett, T. et al. NCBI GEO: archive for functional genomics data sets-update. Nucleic Acids Res, 41, D99-D995 (2013).

4. Bergethon, K. E. et al. Trends in 30-Day Readmission Rates for Patients Hospitalized With Heart Failure: Findings From the Get With The Guidelines-Heart Failure Registry. Circ. Heart Fail, 9, e002594 (2016).

5. Braunwald, E. Heart failure. JACC Heart Failure, 1, 1-20 (2013).

6. Eckhouse, S. R., Jones, J. A. \& Spinale, F. G. Gene targeting in ischemic heart disease and failure: Translational and clinical studies. Biochem. Pharmacol, 85, 1-11 (2013).

7. El-Behi, M. et al. The encephalitogenicity of $\mathrm{T}(\mathrm{H}) 17$ cells is dependent on IL-1- and IL-23-induced production of the cytokine GM-CSF. Nat. Immunol, 12, 568-575 (2011).

8. Elliott, P. Cardiomyopathy. Diagnosis and management of dilated cardiomyopathy., 84, 106 (2000).

9. Eriksson, U. et al. Dendritic cell-induced autoimmune heart failure requires cooperation between adaptive and innate immunity. Nat. Med, 9, 1484-1490 (2003).

10. Frangogiannis, N. G. The mechanistic basis of infarct healing. Antioxid. Redox Signal, 8, 1907-1939 (2006). 
11. Frangogiannis, N. G. \& Entman, M. L. Chemokines in myocardial ischemia. Trends Cardiovasc. Med, $15,163-169$ (2005).

12. Gill, R. G. \& Lin, C. M. Linking innate immunity and chronic antibody-mediated allograft rejection. Curr. Opin. Organ Transplant, 24, 694-698 (2019).

13. Gröschel, C. et al. 2018. CD8 +-T Cells With Specificity for a Model Antigen in Cardiomyocytes Can Become Activated After Transverse Aortic Constriction but Do Not Accelerate Progression to Heart Failure. Front. Immunol. 9, 2665 (2018).

14. Hofmann, U. et al. Activation of CD4 + T Lymphocytes Improves Wound Healing and Survival After Experimental Myocardial Infarction in Mice., 125, 1652-1663 (2012).

15. Jacoby, D. \& McKenna, W. J. Genetics of inherited cardiomyopathy. Eur. Heart J, 33, 296-304 (2012).

16. Hofmann, K., Clauder, A. \& Manz, R. A. Targeting B Cells and Plasma Cells in Autoimmune Diseases. Front. Immunol, 9, 835 (2018).

17. Kazuaki, M. et al. Regulatory $\mathrm{T}$ lymphocytes attenuate myocardial infarction-induced ventricular remodeling in mice. Int. Heart J, 52, 382-387 (2010).

18. Laroumanie, F. et al. CD4(+) T Cells Promote the Transition From Hypertrophy to Heart Failure During Chronic Pressure Overload., 129, 2111-2124 (2014).

19. Lu, M. et al. Th17/Treg imbalance modulates rat myocardial fibrosis and heart failure by regulating LOX expression. Acta Physiol. (Oxf), 230, e13537 (2020).

20. Marty, R. R. et al. MyD88 Signaling Controls Autoimmune Myocarditis Induction., 113, 258-265 (2006).

21. Matthias, N. Myeloid cell contributions to cardiovascular health and disease. Nat. Med, $24,711-720$ (2018).

22. Michowitz, Y. et al. Predictive value of high sensitivity CRP in patients with diastolic heart failure. Int. J. Cardiol, 125, 347-351 (2008).

23. Moro-García, M. A. et al. More intensive CMV-infection in chronic heart failure patients contributes to higher T-lymphocyte differentiation degree. Clin. Immunol, 192, 20-29 (2018).

24. Mosterd, A. et al. The prognosis of heart failure in the general population: The Rotterdam Study. Eur. Heart J, 22, 1318-1327 (2001).

25. Myers, J. M. et al. 2016. Cardiac myosin-Th17 responses promote heart failure in human myocarditis. JCl Insight 1, e85851 (2016).

26. Nevers, T. et al. Th1 effector T cells selectively orchestrate cardiac fibrosis in nonischemic heart failure. J. Exp. Med, 214, 3311-3329 (2017).

27. Ngwenyama, N. et al. CXCR3 regulates CD4 + T cell cardiotropism in pressure overload-induced cardiac dysfunction. JCl Insight, 4, e125527 (2019).

28. Qu, X. K. et al. Prevalence and spectrum of LRRC10 mutations associated with idiopathic dilated cardiomyopathy. Mol. Med. Rep, 12, 3718-3724 (2015). 
29. Rhee, A. \& Lavine, K. New Approaches to Target Inflammation in Heart Failure: Harnessing Insights from Studies of Immune Cell Diversity. Annu. Rev. Physiol, 82, 1-20 (2020).

30. Sager, H. B. et al. Proliferation and Recruitment Contribute to Myocardial Macrophage Expansion in Chronic Heart Failure. Circ. Res, 119, 853-864 (2016).

31. Savic-Radojevic, A. et al. The role of serum VCAM-1 and TNF-a as predictors of mortality and morbidity in patients with chronic heart failure. J. Clin. Lab. Anal, 27, 105-112 (2013).

32. Schultheiss, H. P. et al. Dilated cardiomyopathy. Nat. Rev. Dis. Primers, 5, 32 (2019).

33. Shahid, F., Lip, G. Y. H. \& Shantsila, E. Role of Monocytes in Heart Failure and Atrial Fibrillation. J. Am. Heart Assoc, 7, e007849 (2018).

34. Strassheim, D., Dempsey, E., Gerasimovskaya, E., Stenmark, K. \& Karoor, V. Role of Inflammatory Cell Subtypes in Heart Failure. Journal of Immunology Research 2019, 2164017 (2019).

35. Subramanian, A. et al. Gene set enrichment analysis: A knowledge-based approach for interpreting genome-wide expression profiles. Proc. Natl. Acad. Sci. U S A 102, 15545-15550(2005).

36. Sweet, M. E. et al. Transcriptome analysis of human heart failure reveals dysregulated cell adhesion in dilated cardiomyopathy and activated immune pathways in ischemic heart failure. BMC Genomics, 19, 812 (2018).

37. Tomasoni, D., Adamo, M., Lombardi, C. M. \& Metra, M. Highlights in heart failure. ESC Heart Fail, 6, 1105-1127 (2019).

38. Tousoulis, D. et al. Increased plasma adhesion molecule levels in patients with heart failure who have ischemic heart disease and dilated cardiomyopathy. Am. Heart J, 141, 277-280 (2001).

39. Travers, J. G., Kamal, F. A., Robbins, J., Yutzey, K. E. \& Blaxall, B. C. Cardiac Fibrosis: The Fibroblast Awakens. Circ. Res, 118, 1021-1040 (2016).

40. Wrigley, B., Shantsila, E., Tapp, L. \& Gregory, L. Increased expression of cell adhesion molecule receptors on monocyte subsets in ischaemic heart failure. Thromb. Haemost, 109, 92-100 (2013).

41. Zhang, Y., Bauersachs, J. \& Langer, H. F. Immune mechanisms in heart failure. Eur. J Heart Fail, 19, 1379-1389 (2017).

\section{Figures}




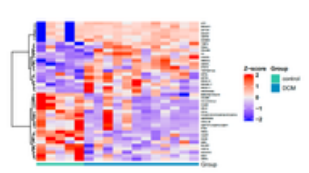

A

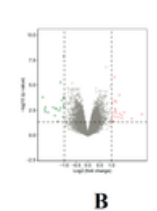

B

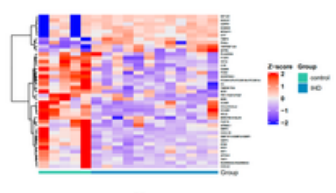

$\mathrm{C}$

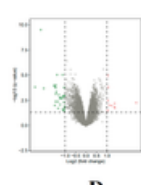

D

\section{ID VS control}
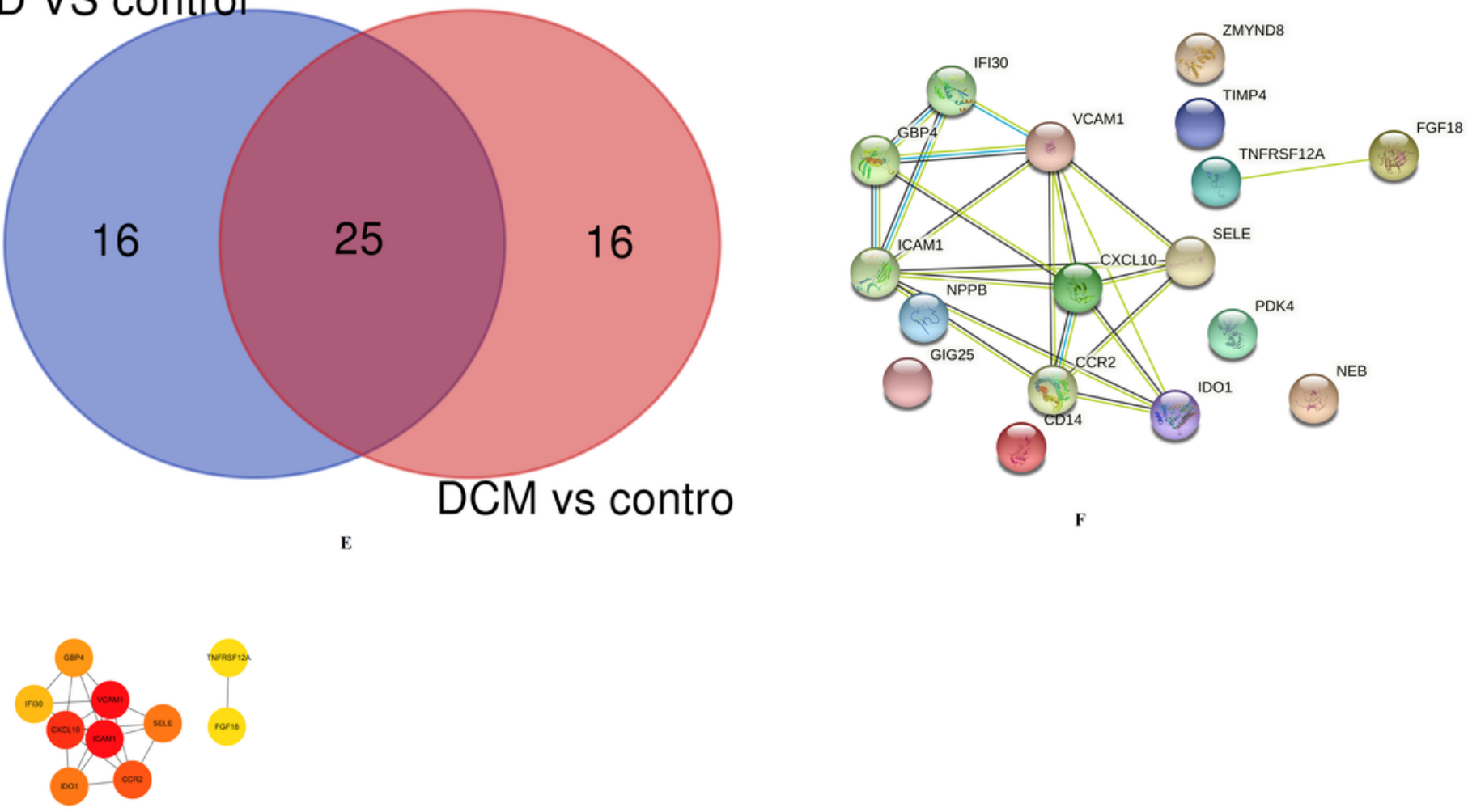

G

Figure 1

(a) Heat map of DEGs screened in myocardial tissue samples of subjects with DCM and controls in the GSE42955 dataset. (b) Volcanogram showing DEG screening of myocardial tissue from subjects with DCM and controls in the GSE42955 dataset. (c) Heat map of DEGs in myocardial tissue samples of subjects with IHD and controls in the GSE42955 dataset. (d) Volcanogram showing DEG screening of myocardial tissue from subjects with IHD and controls in the GSE42955 dataset. (e) Intersection of DEGs in the two cohorts. (f) PPI network for common DEGs. (g) The core function modules of the PPI network. 


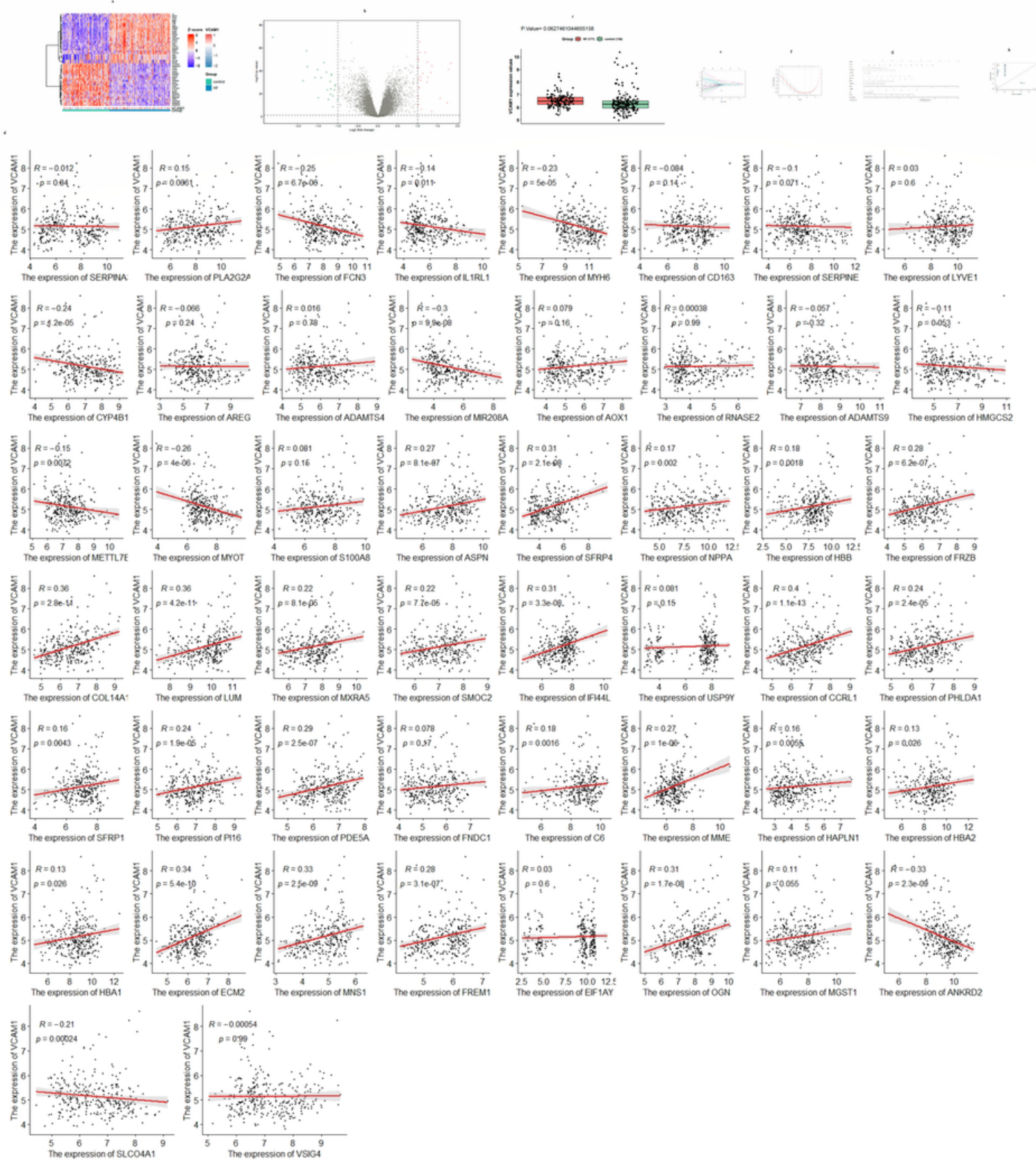

\section{Figure 2}

(a) Heat map of DEG patterns in myocardial tissue from patients with HF compared with controls in the GSE57338 dataset. (b) Volcanogram of DEGs in cardiac tissue from patients with HF compared with controls in the GSE57338 dataset. (c) Box plot showing significantly increased VCAM1 gene expression in patients with HF. (d) Correlation analysis between VCAM1 gene expression and DEGs. (e) LASSO regression was used to select variables suitable for the risk prediction model. (f) Cross-validation of errors between regression models corresponding to different lambda values. $(\mathrm{g})$ Nomogram of the risk model. (h) Calibration curve of the nomogram. (i) Calibration curve in the validation cohort. (j) VCAM1 expression was divided into two groups, and ( $k$ ) risk scores were then compared. 


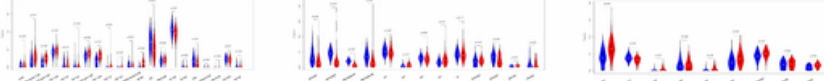
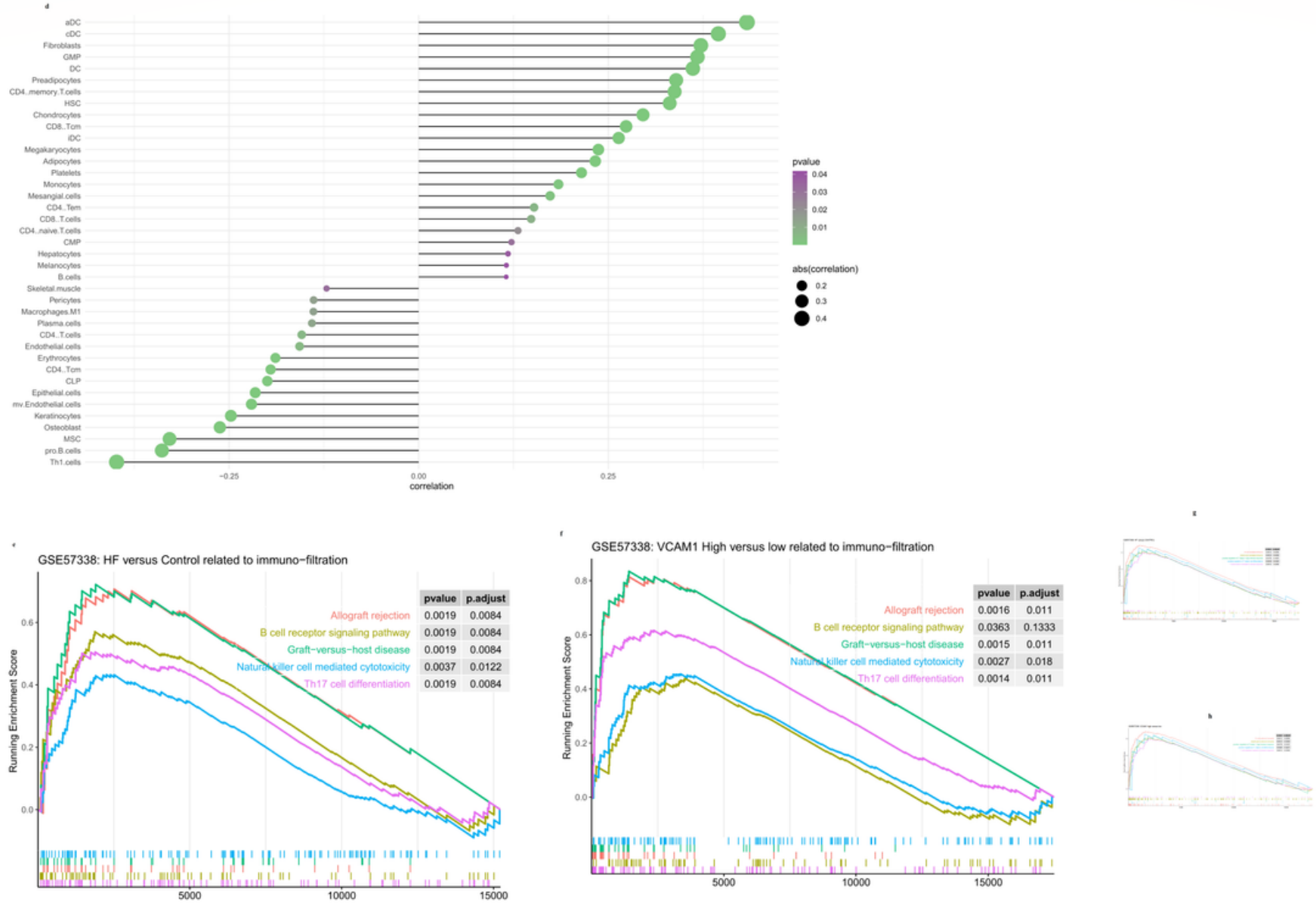

\section{Figure 3}

(a) The degree of lymphocyte immune infiltration in the HF and control groups (red represents samples from failing hearts and blue represents control samples). (b) The degree of myeloid cell immune infiltration in the HF and control groups (red represents samples from failing hearts and blue represents control samples). (c) The degree of stem cell immune infiltration in the HF and control groups (red represents samples from failing hearts and blue represents control samples). (d) Correlation between VCAM1 expression and the infiltration degrees of various cells. (e) Analysis of KEGG pathway enrichment degree between the HF and control groups. (f) Analysis of KEGG pathway enrichment degree between the VCAM1 high- and low-expression groups. (g) Analysis of GO BP enrichment degree between the HF and control groups. (h) Analysis of GO BP enrichment degree between the VCAM1 high- and low-expression groups.

\section{Supplementary Files}


This is a list of supplementary files associated with this preprint. Click to download.

- Supplementaryfigure1.png

- Supplementaryfigure2.png

- GSE5406normalized.csv

- GSE42955normalized.csv

- groupbyVCAM.csv

- dataxs.csv

- dataexternal.csv

- GSE57338normalized.csv

- GSE76701normalized.csv

- immunedeconv.csv

- lowhighGSEAGoBP.csv

- lowhighGSEAKEGG.csv

- HFcontrolgSEAKEGG.csv

- HFcontrolgSEAGoBP.csv 